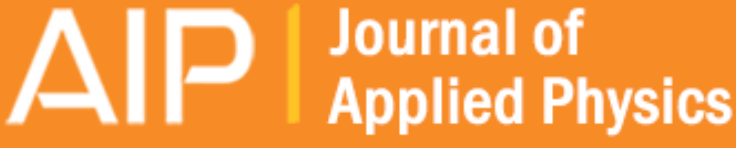

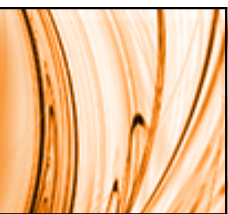

Magnetooptical study of Zeeman effect in Mn modulation-doped InAs/InGaAs/InAIAs quantum well structures

Ya. V. Terent'ev, S. N. Danilov, H. Plank, J. Loher, D. Schuh, D. Bougeard, D. Weiss, M. V. Durnev, S. A.

Tarasenko, I. V. Rozhansky, S. V. Ivanov, D. R. Yakovlev, and S. D. Ganichev

Citation: Journal of Applied Physics 118, 113906 (2015); doi: 10.1063/1.4931154

View online: http://dx.doi.org/10.1063/1.4931154

View Table of Contents: http://scitation.aip.org/content/aip/journal/jap/118/11?ver=pdfcov

Published by the AIP Publishing

\section{Articles you may be interested in}

Magneto-photoluminescence of InAs/InGaAs/InAIAs quantum well structures

Appl. Phys. Lett. 104, 101111 (2014); 10.1063/1.4868644

Strain effects, electronic parameters, and electronic structures in modulation-doped In x Ga 1-x As/In y Al 1-y As coupled step-rectangular quantum wells

J. Appl. Phys. 94, 7621 (2003); 10.1063/1.1631078

Magneto-optical investigations of single self-assembled InAs/InGaAlAs quantum dashes

Appl. Phys. Lett. 82, 2799 (2003); 10.1063/1.1570518

Magnetotransport, magneto-optical, and electronic subband studies in In x Ga 1-x As/In y Al 1-x As modulationdoped strained double quantum wells

J. Appl. Phys. 82, 4388 (1997); 10.1063/1.366165

Identification of room temperature photoluminescence in pseudomorphic modulation-doped

AlGaAs/InGaAs/GaAs quantum wells

J. Appl. Phys. 82, 1345 (1997); 10.1063/1.365908

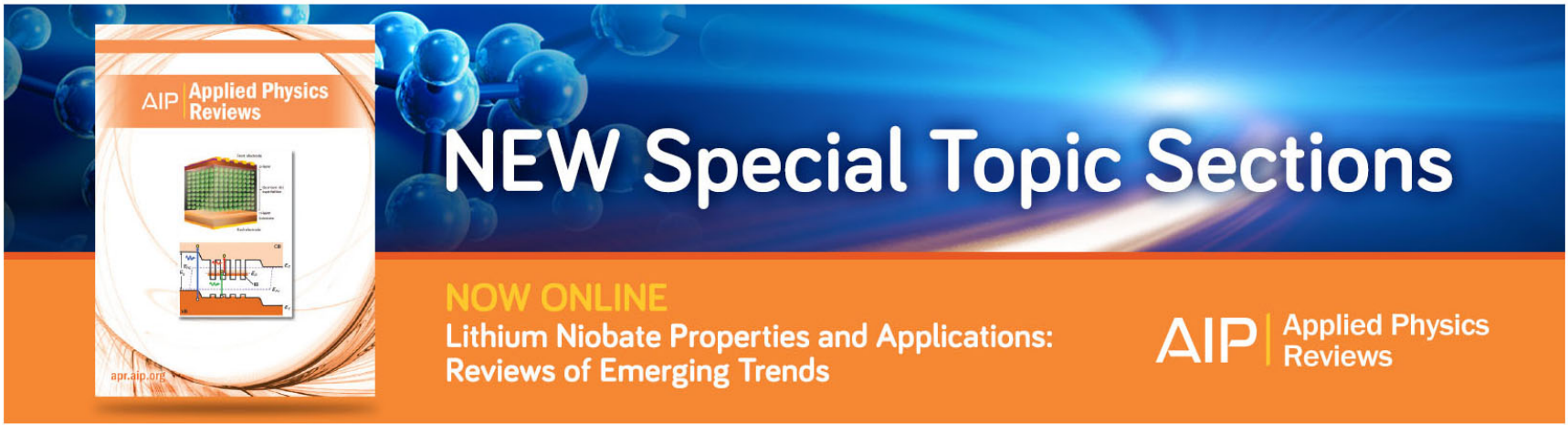




\title{
Magnetooptical study of Zeeman effect in Mn modulation-doped InAs/InGaAs/InAIAs quantum well structures
}

\author{
Ya. V. Terent'ev, ${ }^{1,2}$ S. N. Danilov, ${ }^{1}$ H. Plank, ${ }^{1}$ J. Loher, ${ }^{1}$ D. Schuh, ${ }^{1}$ D. Bougeard, ${ }^{1}$ D. Weiss, ${ }^{1}$ \\ M. V. Durnev, ${ }^{2}$ S. A. Tarasenko, ${ }^{2,3}$ I. V. Rozhansky, ${ }^{2,3}$ S. V. Ivanov, ${ }^{2}$ D. R. Yakovlev, ${ }^{2,4}$ \\ and S. D. Ganichev ${ }^{1}$ \\ ${ }^{1}$ Physics Department, University of Regensburg, 93040 Regensburg, Germany \\ ${ }^{2}$ Ioffe Physical-Technical Institute, 194021 St. Petersburg, Russia \\ ${ }^{3}$ St. Petersburg State Polytechnic University, 195251 St. Petersburg, Russia \\ ${ }^{4}$ Experimentelle Physik 2, Technische Universität Dortmund, 44227 Dortmund, Germany
}

(Received 12 May 2015; accepted 6 September 2015; published online 18 September 2015)

\begin{abstract}
We report on a magneto-photoluminescence (PL) study of Zeeman effect in Mn modulation-doped InAs/InGaAs/InAlAs quantum wells (QW). Two PL lines corresponding to the radiative recombination of photoelectrons with free and bound-on-Mn holes have been observed. In the presence of a magnetic field applied in the Faraday geometry, both lines split into two circularly polarized components. While temperature and magnetic field dependence of the splitting are well described by the Brillouin function, providing an evidence for exchange interaction with spin polarized manganese ions, the value of the splitting exceeds by two orders of magnitude the value of the giant Zeeman splitting estimated for the average Mn density in QW obtained by the secondary ion mass spectroscopy. @ 2015 AIP Publishing LLC. [http://dx.doi.org/10.1063/1.4931154]
\end{abstract}

\section{INTRODUCTION}

Implementation of spintronics concepts requires semiconductor heterostructures with evident magnetic properties. To enhance the interaction between carrier spins and a magnetic field and to achieve ferromagnetic spin ordering doping of materials with magnetic ions is required. ${ }^{1-5}$ Diluted magnetic semiconductors (DMS) based on narrow-gap III-V compounds, and, in particular, InMnAs, are considered to be promising candidates for application due to a relatively high Curie temperature and the strong spin-orbit interaction. ${ }^{6,7}$ While InAs-based DMS systems with strong spin-orbit coupling have been realized and show very interesting magnetotransport ${ }^{8-10}$ and opto-electronic properties, ${ }^{11-15}$ direct measurements of the giant Zeeman splitting by means of polarized magneto-photoluminescence (PL) have not been reported so far. This is primary caused by the fact that the doping of III-V compounds by $\mathrm{Mn}$ atoms generates numerous lattice defects (even if they are partially removed by annealing ${ }^{7}$ ) and, consequently, to a drastic decrease of the radiation efficiency.

In this paper, we report on the observation of circularly polarized magneto-PL from high-quality DMS heterostructures InAs/ $/ \mathrm{In}_{0.75} \mathrm{Ga}_{0.25} \mathrm{As} / \mathrm{In}_{0.75} \mathrm{Al}_{0.25} \mathrm{As}: \mathrm{Mn}$ and systematic study of the Zeeman effect in this system. Our results show that efficient photoluminescence can be excited in such structures with Mn modulation-doped layer introduced in close proximity to InAs QW. The enhanced magnetic properties come from $\mathrm{Mn}$ segregation from modulation-doped barrier, which yields high quality DMS structures as confirmed by quantum Hall experiments. ${ }^{9}$ In zero magnetic field, the low-temperature PL from QW is contributed by two lines separated by $25 \mathrm{meV}$. The analysis shows that the observed PL lines stem from the recombination of free and bound-onMn holes with photoexcited electrons. A magnetic field, applied in the Faraday geometry, results in the splitting of both PL lines into two circular-polarized components with the opposite helicity, reflecting spin splitting of the energy levels in QW. The splitting is strongly temperature dependent and, at low temperatures, saturates with the increase of magnetic field. Both temperature and magnetic field dependences are well fitted by the Brillouin function being typical for the magnetization of DMSs controlled by the exchange interaction of carriers with $\mathrm{Mn}$ ions. However, the value of the detected line splitting exceeds by two orders of magnitude the value of the giant Zeeman splitting estimated for the average Mn density in QW obtained by the secondary ion mass spectroscopy. Possible reasons of this striking observation are discussed.

\section{SAMPLES}

The InAs/In $\operatorname{In}_{0.75} \mathrm{Ga}_{0.25} \mathrm{As} / \mathrm{In}_{0.75} \mathrm{Al}_{0.25} \mathrm{As}$ QW heterostructures investigated were fabricated by molecular beam epitaxy (MBE) on a fully relaxed metamorphic $\mathrm{In}_{x} \mathrm{Al}_{1-\mathrm{x}} \mathrm{As} /$ (001)GaAs compositionally graded buffer where the In content $x$ is increased stepwise from 0.05 to 0.75 over $1 \mu \mathrm{m}$ of layer thickness. Such an approach on structure design enables the fabrication of high quality defect-free and strain relaxed virtual substrates of high indium content that allow an effective collection of photogenerated carriers into the QW. ${ }^{16}$

All experimental samples share the same QW design. Following the band lineups sketched in Fig. 1 an $\operatorname{In}_{0.75} \mathrm{Ga}_{0.25} \mathrm{As}$ shallow QW embedded in between $\operatorname{In}_{0.75} \mathrm{Al}_{0.25}$ As barriers features an asymmetrically inserted and compressively strained InAs channel of $4 \mathrm{~nm}$. The distances between the InAs channel edges and the left and right InAlAs/InGaAs interfaces are $2.5 \mathrm{~nm}$ and $13.5 \mathrm{~nm}$, respectively. Structures are finished with a 36-nm-thick InAlAs cap layer. Manganese containing 

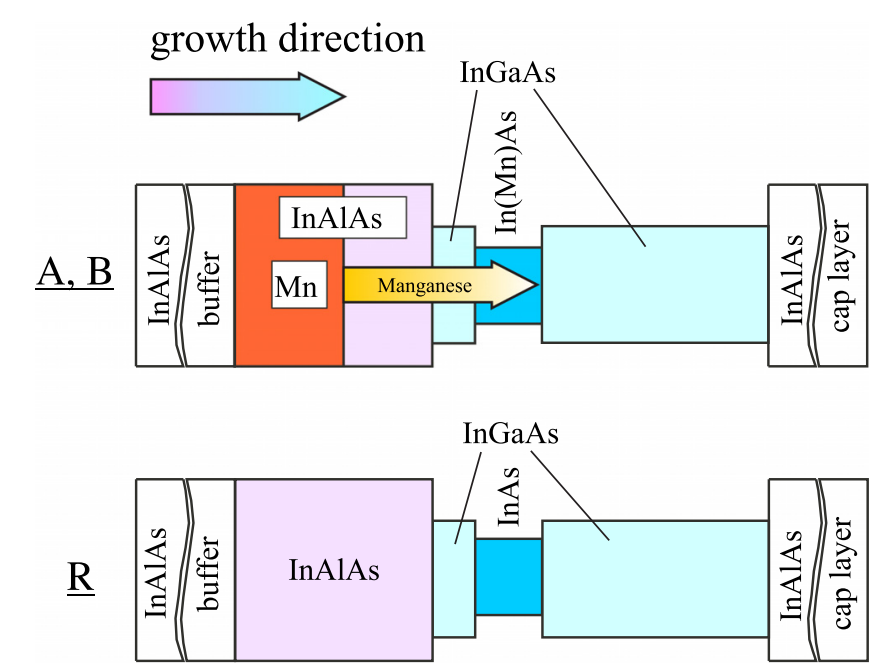

FIG. 1. Band diagrams of samples A, B, and R. GaAs substrate and the InAlAs graded buffer are not shown.

samples possess $7 \mathrm{~nm}$ layers of homogeneously Mn doped $\mathrm{In}_{0.75} \mathrm{Al}_{0.25} \mathrm{As}$ that are inserted on the substrate side of the $\mathrm{QW}$ region ("inverted" doped QWs) and separated from the QW by an $\operatorname{In}_{0.75} \mathrm{Al}_{0.25} \mathrm{As}$ barrier of $5 \mathrm{~nm}$ thickness. Due to a segregation effect during the MBE growth process, a significant amount of manganese resides in the vicinity of the InAs QW. For the sample, referred as A in this paper, $\mathrm{Mn}$ has been inserted for temperature of $\mathrm{Mn}$ effusion cell equal to $852^{\circ} \mathrm{C}$. A dopant concentration not exceeding $n_{\mathrm{Mn}}=2 \times 10^{20} \mathrm{~cm}^{-3}$ that results in a Mn concentration of about two orders of magnitude lower in the InAs QW as revealed by secondary ion mass spectrometry (SIMS). ${ }^{17}$ Hole density in InAs channel determined by Hall effect measurements was $10^{12} \mathrm{~cm}^{-2}$ at $T=4.2 \mathrm{~K}$. While having the same structure design, sample B was grown for substantially lower temperature of $\mathrm{Mn}$ effusion cell $\left(653^{\circ} \mathrm{C}\right)$. The lower cell temperature results in reduction of the segregation and, respectively, magnetic properties. This fact is supported by magnetotransport measurements, in particular, the strong reduction of colossal negative magnetoresistance (not shown), indicating a strong hole localization due to significant Mn concentration in the well. ${ }^{9}$ This effect makes determination of the mobility from Hall-measurements in these structures very challenging. The high quality of the samples becomes, however, obvious at high magnetic fields where the same structures shows well defined quantum Hall steps and Shubnikov de Haas oscillations. ${ }^{9,17,18}$ In addition to Mn doped QW samples A and $\mathrm{B}$, an intentionally undoped structure $\mathrm{R}$ without any Mn implementation was grown to serve as a reference.

\section{EXPERIMENTAL TECHNIQUE}

In our experiments, we have used an experimental setup designed to measure polarization resolved magneto-PL in the infrared (IR) wavelength regime $(2-5 \mu \mathrm{m})$. The setup includes a magnetooptical helium bath cryostat, a laser diode excitation source, an optical polarization system, and a grating or Fourier spectrometer equipped with a nitrogen cooled IR photodetector. Spectra are recorded by using lock-in technique. Magnetic fields up to $6 \mathrm{~T}$ were applied normally to the sample plain and along the wave vector of the emission, i.e., the experiment is done in Faraday geometry. The sample temperature can be varied from 2 up to $300 \mathrm{~K}$. The laser diode, operating in $c w$ mode, emits at wavelength $\lambda=809 \mathrm{~nm}$ and is focused onto a $1 \mathrm{~mm}$ diameter spot at the surface of the sample. The excitation density $W_{\text {exc }}$ can be changed from 0.5 to $20 \mathrm{~W} / \mathrm{cm}^{2}$. The PL emission passes through a polarization system consisting of a quarter wavelength retardation $\mathrm{ZnSe}$ Fresnel rhomb and a linear polarizer having the optical axes crossed at an angle of $\pm 45^{\circ}$ to select $\sigma^{+}$or $\sigma^{-}$polarized light. ${ }^{19}$

\section{EXPERIMENTAL RESULTS}

In all samples, we detected photoluminescence signal, which increases linearly with the excitation density in the explored range $W_{\text {exc }}$ from 0.5 up to $20 \mathrm{~W} / \mathrm{cm}^{2}$. Note that the signal from DMS samples was substantially weaker than that from the reference structure.

The PL band of sample A exhibits two contributions marked in Fig. 2(a) as peak I and peak II, which are separated from each other by about $25 \mathrm{meV}$. In a magnetic field, both peak I and peak II are blue shifted and split into two circularly polarized components, Fig. 2(b). Note, while peak I is $\sigma^{-}$polarized whereas the polarization of peak II is opposite. Splitting reaches a large value up to $6 \mathrm{meV}$, Fig. 3. At low temperatures, the magnetic field dependence of the energy splitting of peak I tends to saturate (see Fig. 4).

Figure 5 shows the magnetic field dependence of the polarization of both PL peaks at different temperatures. At

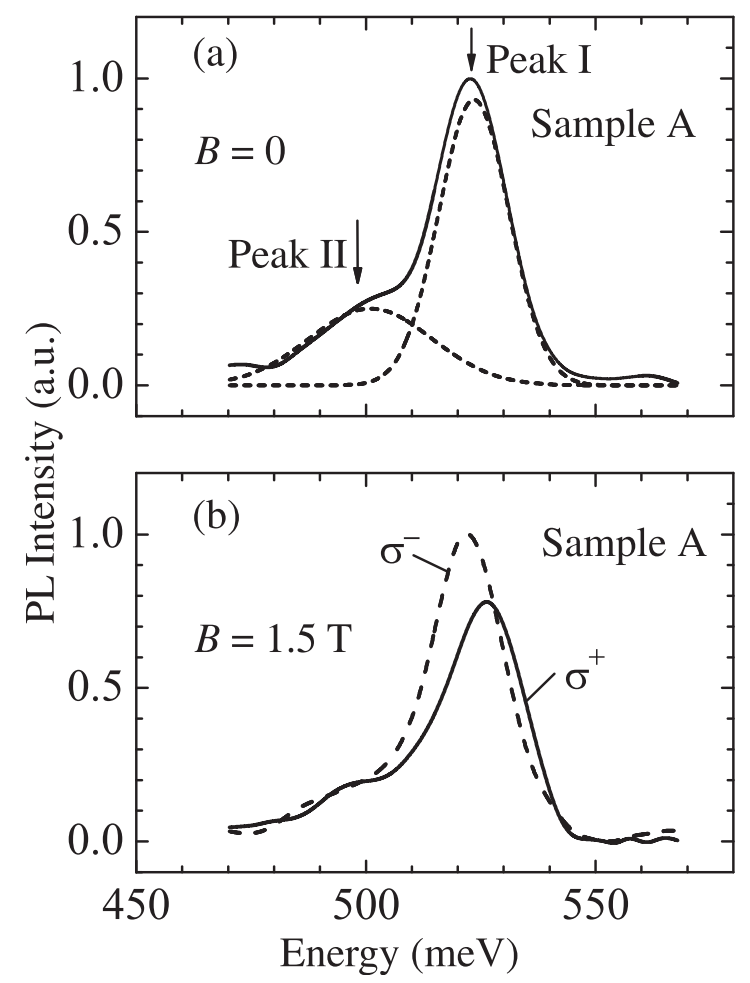

FIG. 2. PL spectra of sample A measured at $T=2 \mathrm{~K}$ and $W_{\text {exc }}=10 \mathrm{~W} / \mathrm{cm}^{2}$. (a) Spectra at zero magnetic field (solid line). Dotted lines shows Lorentzian fit with maxima at energies indicated as peaks I and II. (b) Circularly polarized magneto-PL spectra in the magnetic field of $1.5 \mathrm{~T}$. Solid and dashed lines show the right- and left-handed polarized light, respectively. Plots are normalized to unity at the peak maximum. 


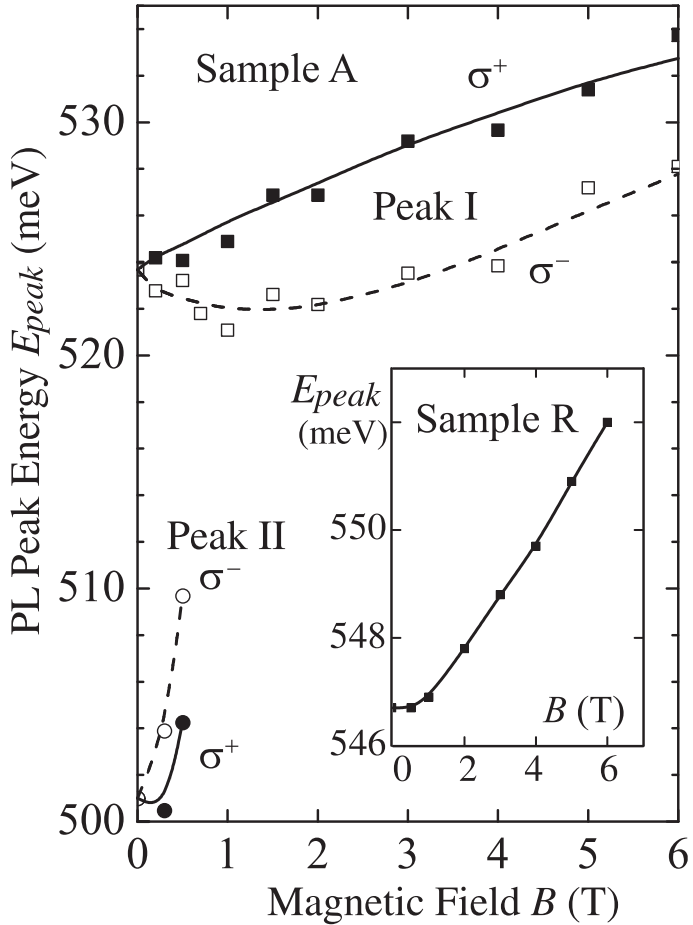

FIG. 3. Energy positions of $\sigma^{+}$and $\sigma^{-}$components of peaks I and II as a function of the magnetic field measured in sample A at $T=2 \mathrm{~K}$ for $W_{e x c}=10 \mathrm{~W} / \mathrm{cm}^{2}$. The inset displays the corresponding dependence obtained in sample R. Lines are a guide for the eyes.

low temperature, the polarization of peak I linearly increases with the magnetic field at small fields and saturates at high fields. The degree of circular polarization of peak I, $P_{\text {circ }}$ $=\frac{I_{+}+I_{-}}{I_{-}-I_{-}} \times 100 \%$, where $I_{+/-}-$is the intensity of $\sigma^{+/-}$polarized emission, reaches $-30 \%$ at $B=6 \mathrm{~T}$. With the temperature increase, the polarization decreases and the saturation vanishes. Behavior of peak II is similar though the polarization at low temperatures cannot be reliably determined due to the disappearance of peak II in the magnetic fields stronger than $1 \mathrm{~T}$.

Sample B also demonstrates two PL lines (Fig. 6(a)). However, peak II is poorly pronounced so that it cannot be analyzed. Peak I demonstrates a blue shift and a strong polarization in the magnetic field ( $P_{\text {circ }}=-40 \%$ at $B=6 \mathrm{~T}$ ). The sign of the polarization is the same as for sample $\mathrm{A}$, but, in contrast to what was observed in sample A, peak I in sample B exhibits no splitting, see Fig. 6(b), within our spectral resolution of $\simeq 0.3 \mathrm{meV}$.

In reference sample R, PL contour consists of a single narrow peak (indicated as peak I) while the second peak (peak II) is absent, Fig. 6(c). Application of a magnetic field results in a blue shift of the PL line and a strong circular polarization which reaches $+54 \%$ in a magnetic field of $6 \mathrm{~T}$ (These data are presented in Ref. 16). Similar to sample $\mathrm{B}$, no energy splitting of the peak has been detected (see Fig. 6(d)).

The large energy spitting and polarization of the peaks I and II measured in sample A, their magnetic field and temperature dependencies, as well as the absence of line splitting in samples $\mathrm{R}$ and $\mathrm{B}$, reveal the important role of the exchange interaction between $\mathrm{Mn}$ ions and carriers confined in QW.

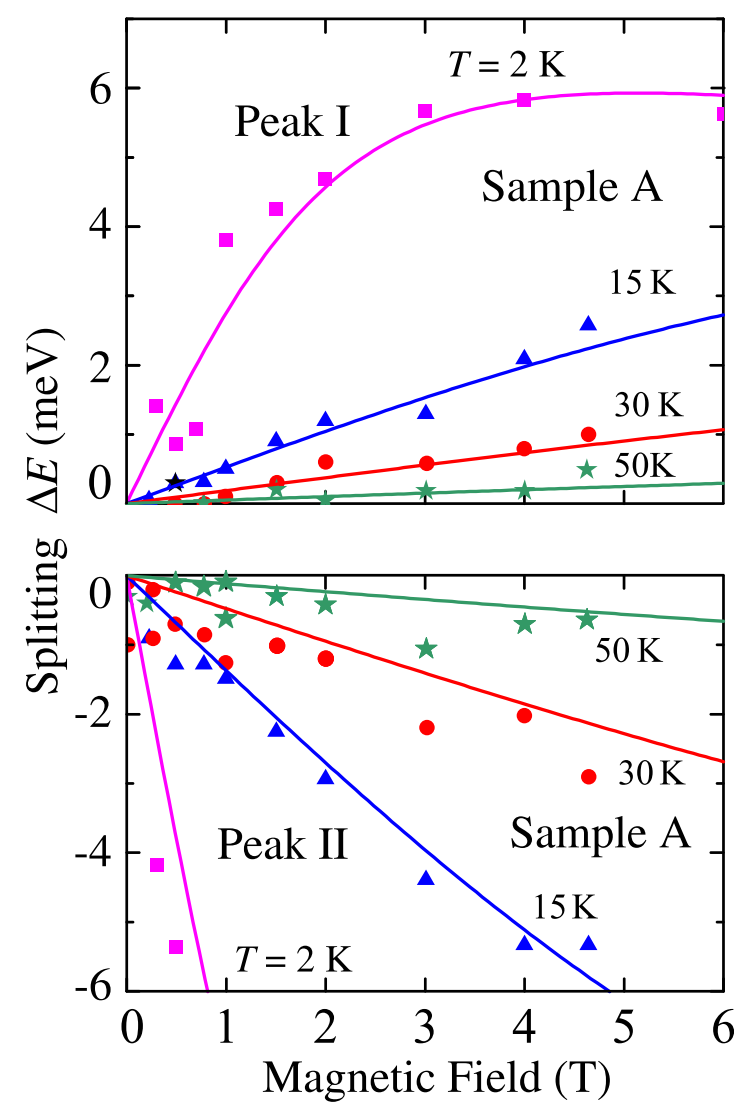

FIG. 4. Magnetic field dependence of the energy splitting $\Delta E$ of peaks I and II, measured in sample $\mathrm{A}$ at different temperatures. $\Delta E$ is defined as $E_{+}-E_{-}$, where $E_{+/-}$is the energy corresponding to the $\sigma^{+/-}$polarized components. Dots represent the experimental data obtained at different temperatures. Lines correspond to the theoretical fit after Eqs. (6) and (8). The effective temperature $T_{\mathrm{Mn}}$ is assumed to be equal to sample temperature $T$, except at $T=2 \mathrm{~K}$, where the best fit corresponds to $T_{\mathrm{Mn}}=3.3 \mathrm{~K}$.

\section{DISCUSSION}

We begin with the origin of the peak I, which is observed in both DMS structures and the reference sample. We ascribe this peak to radiative recombination of electrons and heavy holes occupying the ground levels e1 and hh1, respectively, in the QW. It has been studied in detail in nonmagnetic structures similar to our sample R. ${ }^{16}$ It should be noted that excitonic effects are negligible in our experiments. Indeed, the exciton binding energy is known to be of the order of $1 \mathrm{meV}$ in InAs QWs whereas the Fermi energy due to the large hole concentration in studied Mn-doped samples is much larger. The density of photoelectrons contributing to radiative recombination is far lower and they seem to be localized with binding energies of a few $\mathrm{meV}$. Localization centers can emerge due to inhomogenity of the InAs QW and presence of charged ions. This conclusion is confirmed by the quadratic magnetic field dependence of the PL peak energy clearly detected in the reference sample $\mathrm{R}$ for magnetic fields up to about $3 \mathrm{~T}$, see the inset in Fig. 3. While being superimposed by the Zeeman splitting the quadratic $B$-field dependence is also observed in the DMS structures, see the data for sample A in Fig. 3.

Peak II, red-shifted to peak I and detected in Mn doped structures only, we attribute to optical transitions of conduction 


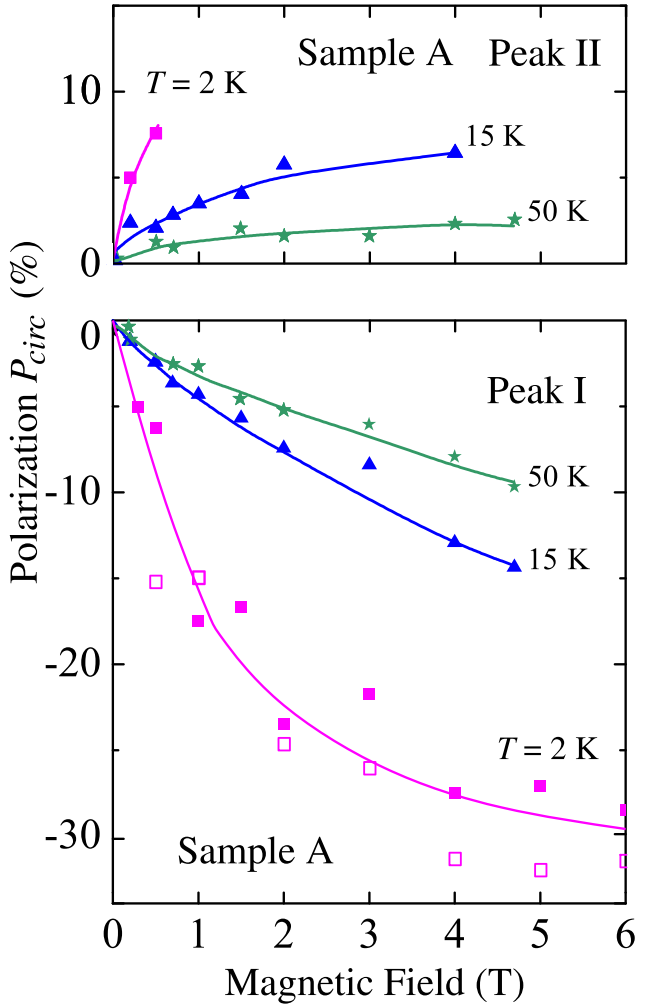

FIG. 5. Polarization degree $P_{\text {circ }}$ of peaks I and II measured in sample A at $T=2 \mathrm{~K}$ for $W_{\text {exc }}=10 \mathrm{~W} / \mathrm{cm}^{2}$ (solid symbols) and $5 \mathrm{~W} / \mathrm{cm}^{2}$ (open squares). Lines are a guide for the eyes.

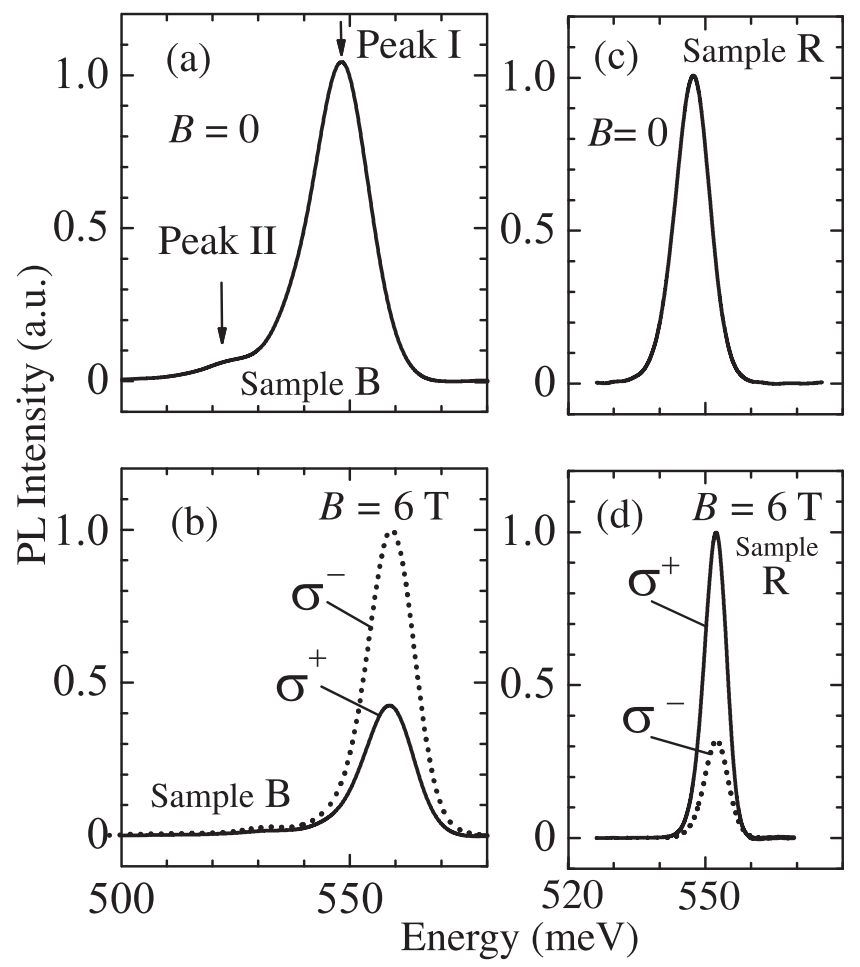

FIG. 6. PL spectra of sample $\mathrm{B}$ and $\mathrm{R}$ measured at $T=2 \mathrm{~K}$ for $W_{\text {exc }}$ $=10 \mathrm{~W} / \mathrm{cm}^{2}$. (a) and (b) Spectra obtained for sample B in a magnetic field of 0 and $6 \mathrm{~T}$, respectively. (c) and (d) The same dependences for sample R. Solid and dashed lines show the right- and left-handed polarized light, respectively. Plots are normalized to unity at the peak maximum. electrons to the acceptor level of Mn ions, embedded in the QW. Indeed, a shallow manganese impurity band lying $23 \mathrm{meV}$ below the InAs valence band edge was revealed in bulk (In,Mn)As. ${ }^{20}$ Using this value as a reference for the $\mathrm{Mn}$ shallow acceptor binding energy in bulk InAs, we have calculated the binding energy $E_{\mathrm{Mn}}$ in the QW. For that we followed the method suggested in Ref. 21 and treated the acceptor in zero-range potential approximation. Within the approach the bound state wave function $\Psi$ satisfies the Schrödinger equation

$$
H_{L} \Psi=E_{\mathrm{Mn}} \Psi+2 \pi \delta\left(\mathbf{r}-\mathbf{r}_{0}\right) \Psi_{0},
$$

where $H_{L}$ is the Luttinger Hamiltonian, $\mathbf{r}_{0}$ is the acceptor position. The 4-component function $\Psi_{0}$ is to be found from the boundary conditions. At the impurity site $\mathbf{r}_{0}$, the boundary condition for the angular averaged wavefunction $\bar{\Psi}$ reads

$$
\left.\bar{\Psi}\right|_{\mathbf{r} \rightarrow \mathbf{r}_{0}}=\left(\frac{1}{\left|\mathbf{r}-\mathbf{r}_{0}\right|}-\alpha\right) \Psi_{0}+o\left(\left|\mathbf{r}-\mathbf{r}_{0}\right|\right),
$$

where $\alpha$ is the parameter of the impurity attractive potential strength in the zero-range potential model. At the QW boundaries, all components of $\Psi$ were set to zero, thus implying the boundary conditions for the infinitely deep QW. Setting the QW width to infinity enabled us to find $\alpha$ from Eqs. (1) and (2) with $E_{\mathrm{Mn}}$ being set to the Mn binding energy for the bulk. Restoring the QW width to its experimental value we obtained the binding energy $E_{\mathrm{Mn}}$ from Eqs. (1) and (2) with known $\alpha$. The calculation shows that for the system studied, containing the $4 \mathrm{~nm}$ wide $\mathrm{QW}, E_{\mathrm{Mn}}$ is practically the same as that in bulk InAs, though in narrower QWs, it decreases swiftly. The particular value $E_{\mathrm{Mn}} \simeq 25 \mathrm{meV}$ obtained for $\mathrm{Mn}$ ion in the center of the $4 \mathrm{~nm}$ QW is in good agreement with the experimentally observed red shift of peak II relative to peak I. Attribution of peak II to the presence of $\mathrm{Mn}$ in QW is consistent with the fact that peak II is extremely weak in sample B having lower Mn content. Acceptor levels can be also probed by another technique, e.g., electron paramagnetic resonance. ${ }^{22,23}$

We now turn to the case of an applied magnetic field. The magneto-PL of the manganese-free structure $\mathrm{R}$ was studied in detail in Ref. 16. Here, we give only a brief summary of the main results, which are important for the further discussion. The application of a magnetic field results in the spin splitting of both, conduction and valence bands. Optical recombination processes of the electron $|e 1,+1 / 2\rangle$ with the hole $|h h 1,-3 / 2\rangle$ or the electron $|e 1,-1 / 2\rangle$ and the hole $|h h 1,+3 / 2\rangle$ are accompanied by the emission of $\sigma^{-}$- and $\sigma^{+}$-polarized photons, respectively, that are detected in the Faraday geometry of the experiment. The strong $\sigma^{+}$circular polarization of the magneto-PL peak stems from the spin polarization of the nonequilibrium holes, characterized by a faster relaxation to the ground Zeeman level in comparison with electrons. The same polarization is observed in structures with degenerate 2DEG, where the different electron spin states are equally populated. ${ }^{16}$ The absence of the splitting of the PL line into polarized components of different helicity was explained by the nearly equal magnitude of the electron and hole Lande factors in the system. ${ }^{16}$ 
Within the suggested model, the polarization must invert its sign to $\sigma^{-}$if we change type of conductivity of the QW from $n$-type to strong $p$-type. Indeed, at the condition of equally populated spin levels in the valence band in $p$-type samples, polarization is determined by electrons and recombination of electron at the ground state $|e 1,+1 / 2\rangle$ (for negative electron $g$-factor) with the hole $|h h 1,-3 / 2\rangle$ must dominate. This process is accompanied by the emission of $\sigma^{-}$-polarized photons. Samples A and B contain degenerated 2DHG; thus, $\sigma^{-}$polarization of peak I detected from these samples is consistent with the model. Zero splitting of circular polarized PL components in sample B should be obviously attributed to vanishingly small Mn content in the InAs QW.

In contrast to sample B, sample A provides clear evidence that free carriers interact via exchange with $\mathrm{Mn}$ ions embedded in the InAs QW. Indeed, application of a magnetic field results in a large splitting of peak I into circularly polarized components (Fig. 4) which is accurately described by the Brillouin function in a wide temperature range (fitting details are given below). The splitting tends to saturate in magnetic fields higher than $3-4 \mathrm{~T}$ at the lowest achieved temperature of $2 \mathrm{~K}$ and decreases rapidly if temperature is raised above $\sim 10 \mathrm{~K}$. The polarization dependence on magnetic field and temperature is similar to that of the splitting, Fig. 5.

To clarify a possible influence of photoexcitation induced carrier heating on polarization degree, we measured $P_{\text {circ }}$ for different excitation power varying in the range from 5 to $10 \mathrm{~W} / \mathrm{cm}^{2}$. Figure 5 (bottom panel) shows magnetic field dependence of $P_{\text {circ }}$ obtained at excitation power $W_{e x c}=5$ to $10 \mathrm{~W} / \mathrm{cm}^{2}$ for peak I in sample A at $T=2 \mathrm{~K}$. This and other data reveal that varying the excitation power in the examined range does not demonstrate any noticeable effect on PL polarization even at low temperatures. Thus, the contribution of light heating can be considered as negligible.

We note that all the investigated structures show a polarization degree which is considerably below $100 \%$ even at $T=2 \mathrm{~K}$ and $B=6 \mathrm{~T}$ although the dependence $P_{\text {circ }}$ vs $B$ tends to saturate. This fact can be ascribed to the interplay between the short lifetime of photogenerated carriers compared to the spin relaxation time. In the particular case of $p$-type QWs, where the holes are unpolarized and the PL polarization is determined solely by the electron spin polarization, the PL polarization is given by $P_{c}=-2 S_{e}^{(0)} \tau_{r} /\left(\tau_{r}+\tau_{s}\right)$. Here, $S_{e}^{(0)}$ is the average electron spin in thermal equilibrium, and $\tau_{r}$ and $\tau_{s}$ are the electron lifetime and spin relaxation time, respectively.

Now we turn to analysis of experimental data in the frame of common approach used for description of exchange interaction in DMS. As addressed above, peak II is attributed to the optical transitions between photoelectrons and holes bound to Mn ions (see Fig. 7), which can be schematically described as

$$
\mathrm{e}+(\mathrm{h}+\mathrm{Mn}) \rightarrow \gamma+\mathrm{Mn}
$$

where the symbols e, $\mathrm{h}$, and $\gamma$ denote an electron, hole, and photon, respectively. Mn ion and the hole bound to Mn are antiferromagnetically coupled. In the magnetic field, the bound-hole-Mn complex gets polarized in such a way that the hole spin points along the field direction. Recombination of spin polarized holes with electrons leads to $\sigma^{+}$circular polarization of peak II.

Due to the strong $p$ - $d$ exchange coupling, the ground state of the bound-hole-Mn complex is described by the total angular momentum $J=1$ and is three-fold degenerate in the projection $J_{z}$ at zero magnetic field. ${ }^{24}$ A conduction-band electron, in contrast, has spin $s_{e}=1 / 2$, and each state is two-fold degenerate. Thus, there are six different initial states for the processes described by Eq. (3). The final electron state of $\mathrm{Mn}$ is six-fold degenerate in the projections of the Mn spin $S_{\mathrm{Mn}}=5 / 2$. The allowed optical transitions emitting $\sigma^{+}$-polarized radiation (labeled by the index $k=1 \ldots 6$ ) and their relative intensities $C_{k}$ are summarized in Table I. The transitions allowing the emission of $\sigma^{-}$-polarized photons can be obtained from Table I by simultaneous inverting the sign of $J_{z}, s_{e, z}$ and $S_{\mathrm{Mn}, z}$.

In an external magnetic field, the emission line splits into 12 components, each of them being either $\sigma^{+}$- or $\sigma^{-}$-polarized. The corresponding Zeeman shifts of the $\sigma^{+}$-polarized components are listed in Table I. In a real QW

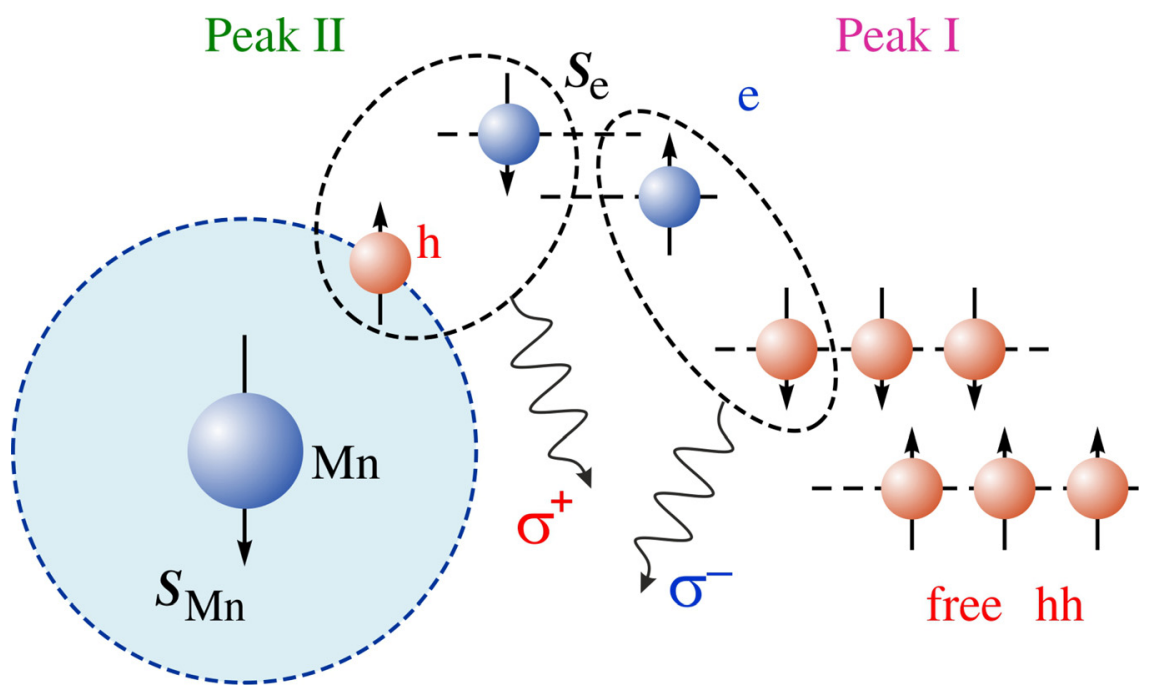

FIG. 7. Sketch of the optical transitions contributing to peaks I and II in magnetic field. Only transitions contributing to dominant circularly polarized components of peaks I and II are depicted. 
TABLE I. Optical transitions between conduction-band electrons and holes bound to Mn with emission of $\sigma^{+}$-polarized photons. $\Delta_{e}, \Delta_{1}$, and $\Delta_{\mathrm{Mn}}$ are the Zeeman splitting of electron states, hole-Mn complex, and Mn ion, respectively.

\begin{tabular}{ccccc}
\hline \hline$k$ & $\begin{array}{c}\text { Initial state } \\
J_{z}, s_{e, z}\end{array}$ & $\begin{array}{c}\text { Final state } \\
S_{\mathrm{Mn}, z}\end{array}$ & $\begin{array}{c}\text { Relative } \\
\text { rate } C_{k}\end{array}$ & $\begin{array}{c}\text { Zeeman shift } \\
\Delta_{k}\end{array}$ \\
\hline 1 & $-1,-1 / 2$ & $-5 / 2$ & $1 / 2$ & $-\Delta_{e} / 2-\Delta_{1}+5 \Delta_{\mathrm{Mn}} / 2$ \\
2 & $-1,+1 / 2$ & $-3 / 2$ & $1 / 10$ & $\Delta_{e} / 2-\Delta_{1}+3 \Delta_{\mathrm{Mn}} / 2$ \\
3 & $0,-1 / 2$ & $-3 / 2$ & $1 / 5$ & $-\Delta_{e} / 2+3 \Delta_{\mathrm{Mn}} / 2$ \\
4 & $0,+1 / 2$ & $-1 / 2$ & $1 / 10$ & $\Delta_{e} / 2+\Delta_{\mathrm{Mn}} / 2$ \\
5 & $+1,-1 / 2$ & $-1 / 2$ & $1 / 20$ & $-\Delta_{e} / 2+\Delta_{1}+\Delta_{\mathrm{Mn}} / 2$ \\
6 & $+1,+1 / 2$ & $+1 / 2$ & $1 / 20$ & $\Delta_{e} / 2+\Delta_{1}-\Delta_{\mathrm{Mn}} / 2$ \\
\hline \hline
\end{tabular}

structure, however, individual components may not be spectrally resolved due to considerable inhomogeneous broadening. Therefore, the measured disorder-broadened spectrum of the $\sigma^{+}$-polarized PL is given by

$$
I_{+}(\hbar \omega)=\sum_{k=1}^{6} C_{k} f_{k} D\left(\hbar \omega-\Delta_{k}\right)
$$

where $f_{k}$ is the population of the initial state with the index $k$ and $D(\hbar \omega)$ is the PL contour at zero magnetic field. To first order in the Zeeman splitting, Eq. (4) is equivalent to

$$
I_{+}(\hbar \omega) \approx D(\hbar \omega) \sum_{k} C_{k} f_{k}-D^{\prime}(\hbar \omega) \sum_{k} C_{k} \Delta_{k} f_{0},
$$

where $D^{\prime}(\hbar \omega)=d D(\hbar \omega) / d \hbar \omega$ and $f_{0}$ is the population at zero magnetic field, identical for all initial states. Equation (5) describes that the PL contour $I_{+}(\hbar \omega)$ is spectrally shifted with respect to the PL contour at zero field by $\sum_{k} C_{k} \Delta_{k} / \sum_{k} C_{k}$. Similarly, the $\sigma^{-}$-polarized PL contour is shifted in the opposite direction by the same value. For the particular case of the optical transitions listed in Table I, the effective Zeeman shift between the broadened PL lines of opposite helicity is given by

$$
\Delta E_{\mathrm{II}}=-\Delta_{1}-\frac{1}{2} \Delta_{e}+\frac{5}{2} \Delta_{\mathrm{Mn}}
$$

The Zeeman splitting of the hole-Mn complex, Mn ion, and conduction-band electron have the form

$$
\begin{aligned}
& \Delta_{1}=g_{1} \mu_{\mathrm{B}} B_{z}, \quad \Delta_{\mathrm{Mn}}=g_{0} \mu_{\mathrm{B}} B_{z}, \\
& \Delta_{e}=g_{e} \mu_{\mathrm{B}} B_{z}+a \mathrm{~B}_{1}\left(\frac{g_{1} \mu_{\mathrm{B}} B_{z}}{k_{\mathrm{B}} T_{\mathrm{Mn}}}\right),
\end{aligned}
$$

where $g_{1}$ is the $g$-factor of the hole-Mn complex, $g_{0}=2$ is the $\mathrm{Mn} g$-factor, $g_{e}$ is the intrinsic electron $g$-factor, $\mu_{\mathrm{B}}$ is the Bohr magneton, $T_{\mathrm{Mn}}$ is the effective temperature of the $\mathrm{Mn}$ spins, and $\mathrm{B}_{1}(x)=2 \sinh x /(1+2 \cosh x)$ is the Brillouin function of the momentum $J=1$. The second contribution to $\Delta_{e}$ describes the splitting due to exchange interaction between conduction-band electrons and hole-Mn complexes, parameter $a$ depends on the exchange interaction strength and complex concentration.

The PL peak I is attributed to the optical transitions between electrons and heavy holes confined in the QW (see Fig. 7). The sign of the PL circular polarization in a magnetic field is largely determined by the spin polarization of electrons since the thermal spin polarization of holes is low in $p$-doped structures. The electron spin polarization can occur due to thermal population of spin-split states as well as spin-dependent extraction of electrons caused by other recombination channels, ${ }^{25}$ e.g., radiative transitions contributing to peak II. The spectral shift between the polarized lines of opposite helicity in a magnetic field is determined by the Zeeman splitting of both, conduction and valence bands, and is given by

$$
\Delta E_{\mathrm{I}}=-\Delta_{e}+\Delta_{h h},
$$

where $\Delta_{h h}$ is the Zeeman splitting of the heavy-hole subband

$$
\Delta_{h h}=g_{h h} \mu_{\mathrm{B}} B_{z}+b \mathrm{~B}_{1}\left(\frac{g_{1} \mu_{\mathrm{B}} B_{z}}{k_{\mathrm{B}} T_{\mathrm{Mn}}}\right) .
$$

Here, $b$ is the parameter describing the strength of the exchange interaction between free holes and hole-Mn complexes.

Peak I and its splitting in magnetic fields is well observed in experiment. In the regime of small magnetic fields, $\Delta E_{\mathrm{I}}$ depends linearly on the magnetic field and has two contributions: one is temperature independent and the other one scales as $1 / T_{\mathrm{Mn}}$. Fitting the experimental data presented in Fig. 4 by Eq. (8) yields $(b-a) g_{1} \approx 24 \mathrm{meV}$ and $g_{e}-g_{h h} \approx 2.6$. The small absolute value of $g_{e}-g_{h h}$ is in agreement with negligible splitting of the PL line in QW structures without magnetic impurities. ${ }^{16}$

We note that at temperatures $T<5 \mathrm{~K}$, PL spectra are weakly sensitive to the sample temperature. This indicates that the effective $\mathrm{Mn}$ temperature $T_{\mathrm{Mn}}$ is higher then the nominal sample temperature due to heating by radiation. In particular, the best agreement between the experimental curve measured at $2 \mathrm{~K}$ and theory is obtained for $T_{\mathrm{Mn}} \approx 3.3 \mathrm{~K}$. Peak II is less pronounced and disappears in high magnetic fields; therefore, its treatment is less reliable. Fitting the data on the Zeeman splitting of peak II by Eq. (6) yields $a g_{1} \approx-126 \mathrm{meV}$ and $2 g_{1}+g_{e} \approx-4.6$.

The saturation of the splitting at higher magnetic fields is determined by $g_{1}$. The best fit of the experimental data for peak I using $T_{\mathrm{Mn}}=3.3 \mathrm{~K}$ is obtained for $g_{1} \approx 3.5$. This value is in agreement with the theoretical calculation and experimental measurements of the $g$-factor of a bound-hole-Mn complex in GaAs crystals $\left(g_{1} \approx 2.7\right){ }^{24,26}$ Taking this $g_{1}$ value, all other parameters can be estimated as $a \approx-36 \mathrm{meV}$, $b \approx-29 \mathrm{meV}, g_{e} \approx-11.6$, and $g_{h h} \approx-14.2$ for the $\mathrm{QW}$ structure under study. The large absolute value and the negative sign of the electron $g$-factor is in agreement with the $g$-factor in bulk InAs: $g_{e} \approx-15 .^{27}$ The negative sign of $b$ is in agreement with the antiferromagnetic behavior of the $p$-d exchange interaction. The determined negative sign of the parameter $a$, which describes the exchange interaction between the conduction-band electron and bound-hole-Mn complex, may be caused by the electron-hole exchange interaction and is discussed in Ref. 28.

While, the above analysis describes all qualitative features of experiments on $\mathrm{Mn}$ doped samples the observed 
splitting of the PL lines in sample A exceeds by two orders of magnitude the one estimated using $s-d$ and $p-d$ exchange-coupling constants for bulk InMnAs, $\alpha$ and $\beta$ of order of $-1 \mathrm{eV}$ and $0.5 \mathrm{eV}$, respectively. ${ }^{11}$ The origin of this discrepancy remains unclear and needs further study. A possible reason for the enhanced Zeeman splitting observed in the experiment might be the formation of Mn-enriched areas due to inhomogeneous Mn distribution in the plane of the QW. It is reasonable to expect that in this case the dominant contribution to the PL is associated with carriers localized in these areas. This would result in a stronger local exchange interaction as compared to that estimated from the average Mn concentration. Indeed, even in the case of high quality metamorphic buffer layers used in our structures the density of extended defects (threading dislocations) lies around $10^{6} \mathrm{~cm}^{-2}$, maximum $10^{7} \mathrm{~cm}^{-2}$. Due to well-known phenomenon-enhanced metal diffusion and accumulation along the threading dislocations in III-V semiconductors ${ }^{29,30}-\mathrm{Mn}$ atoms can accumulate around the residual threading dislocations propagating into the QW. Thus, their local concentration in the InAs QW can exceed the average level of segregated Mn concentration $\sim 10^{18} \mathrm{~cm}^{-3}$ and may in principle be high enough to create the regions of InMnAs with Mn content on the order of $1 \%$. It is reasonable to expect that the dominant contribution to the recombination is associated with the localized charge carriers in these regions. The exchange interaction between charge carriers and $\mathrm{Mn}$ ions in these areas is considerably enhanced comparing to the areas where $\mathrm{Mn}$ ions are distributed homogeneously resulting in larger values of exchange constants.

However, justification of this mechanism needs challenging morphology studies, e.g., by space resolved microphotoluminescence in magnetic field.

\section{SUMMARY}

Our results show that efficient photoluminescence can be excited in InAs/InGaAs/InAlAs heterostructures with $\mathrm{Mn}$ modulation doped layer introduced in close proximity to InAs quantum well. Applying circular polarized magneto-PL we revealed that "inverted" doped QWs structures with Mn inserted at high temperature of $\mathrm{Mn}$ effusion cell equal $\left(852^{\circ} \mathrm{C}\right)$ demonstrate giant Zeeman effect resulting in a high degree of spin levels splitting being up to $6 \mathrm{meV}$ for $T=2 \mathrm{~K}$ and magnetic field $B \geq 4 \mathrm{~T}$. The functional behavior of the PL line splitting resulting from the Zeeman effect, such as its temperature and magnetic field dependencies, is well described by the Brillouin function, providing an evidence for exchange interaction with spin polarized manganese ions. However, the value of the splitting exceeds by two orders of magnitude the one estimated in the frame of common approach to the description of exchange interaction in DMS assuming homogeneous distribution of $\mathrm{Mn}$ ions in the well. While this surprising finding could be ascribed to local Mn accumulation along threading dislocations its origin requires further study. The inhomogeneous distribution of $\mathrm{Mn}$ in the plane of the QW could be investigated by micro-PL technique with high space resolution.

\section{ACKNOWLEDGMENTS}

Financial support by DFG (SFB 689 and ICRC-TRR 160), the Russian Foundation for Basic Research, RF President Grant No. MD-3098.2014.2, the "Dynasty" foundation and EU project SPANGL4Q is gratefully acknowledged.

${ }^{1}$ Spin Physics in Semiconductors, edited by M. I. Dyakonov (Springer, 2008).

${ }^{2}$ Spintronics (Semiconductors and Semimetals), edited by T. Dietl, D. D. Awschalom, M. Kaminska, and H. Ohno (Academic Press, 2009).

${ }^{3}$ Introduction to the Physics of Diluted Magnetic Semiconductors, edited by J. Kossut and J. A. Gaj (Springer, 2011).

${ }^{4}$ Handbook of Spin Transport and Magnetism, edited by E. Y. Tsymbal and I. Zutic (Chapman and Hall, 2011).

${ }^{5}$ T. Jungwirth, J. Wunderlich, V. Novak, K. Olejnik, B. L. Gallagher, R. P. Campion, K. W. Edmonds, A. W. Rushforth, A. J. Ferguson, and P. Nemec, Rev. Mod. Phys. 86, 855 (2014).

${ }^{6}$ T. Dietl, Nature Mat. 9, 965 (2010).

${ }^{7}$ T. Schallenberg and H. Munekata, Appl. Phys. Lett. 89, 042507 (2006).

${ }^{8}$ H. Ohno, H. Munekata, T. Penney, S. von Molnár, and L. L. Chang, Phys. Rev. Lett. 68, 2664 (1992).

${ }^{9}$ U. Wurstbauer, C. Śliwa, D. Weiss, T. Dietl, and W. Wegscheider, Nature Phys. 6, 955 (2010).

${ }^{10}$ B. Rupprecht, W. Krenner, U. Wurstbauer, Ch. Heyn, T. Windisch, M. A. Wilde, W. Wegscheider, and D. Grundler, J. Appl. Phys. 107, 093711 (2010).

${ }^{11}$ A. Zudov, J. Kono, Y. H. Matsuda, T. Ikaida, N. Miura, H. Munekata, G. D. Sanders, Y. Sun, and C. J. Stanton, Phys. Rev. B 66, 161307 (2002).

${ }^{12}$ S. D. Ganichev, S. A. Tarasenko, V. V. Bel'kov, P. Olbrich, W. Eder, D. R. Yakovlev, V. Kolkovsky, W. Zaleszczyk, G. Karczewski, T. Wojtowicz, and D. Weiss, Phys. Rev. Lett. 102, 156602 (2009).

${ }^{13}$ V. V. Bel'kov and S. D. Ganichev, Semicond. Sci. Technol. 23, 114003 (2008).

${ }^{14}$ P. Olbrich, C. Zoth, P. Lutz, C. Drexler, V. V. Bel'kov, Ya. V. Terent'ev, S. A. Tarasenko, A. N. Semenov, S. V. Ivanov, D. R. Yakovlev, T. Wojtowicz, U. Wurstbauer, D. Schuh, and S. D. Ganichev, Phys. Rev. B 86, 085310 (2012).

${ }^{15}$ G. A. Khodaparast, Y. H. Matsuda, D. Saha, G. D. Sanders, C. J. Stanton, H. Saito, S. Takeyama, T. R. Merritt, C. Feeser, B. W. Wessels, X. Liu, and J. Furdyna, Phys. Rev. B 88, 235204 (2013).

${ }^{16}$ Ya. V. Terent'ev, S. N. Danilov, J. Loher, D. Schuh, D. Bougeard, D. Weiss, M. V. Durnev, S. A. Tarasenko, M. S. Mukhin, S. V. Ivanov, and S. D. Ganichev, Appl. Phys. Lett. 104, 101111 (2014).

${ }^{17}$ U. Wurstbauer, M. Soda, R. Jakiela, D. Schuh, D. Weiss, J. Zweck, and W. Wegscheider, J. Cryst. Growth 311, 2160 (2009).

${ }^{18}$ U. Wurstbauer and W. Wegscheider, Phys. Rev. B. 79, 155444 (2009).

${ }^{19}$ S. D. Ganichev, Petra Schneider, V. V. Bel'kov, E. L. Ivchenko, S. A. Tarasenko, W. Wegscheider, D. Weiss, D. Schuh, B. N. Murdin, P. J. Phillips, C. R. Pidgeon, D. G. Clarke, M. Merrick, P. Murzyn, E. V. Beregulin, and W. Prettl, Phys. Rev. B 68, 081302 (2003).

${ }^{20}$ P. T. Chiu, A. J. Blattner, S. J. May, and B. W. Wessels, Physica B 344, 379 (2004).

${ }^{21}$ N. S. Averkiev, A. E. Zhukov, Yu. L. Ivanov, P. V. Petrov, K. S. Romanov, A. A. Tonkikh, V. M. Ustinov, and G. E. Tsyrlin, Semiconductors 38, 217 (2004).

${ }^{22}$ H. Kaftelen, K. Ocakoglu, R. Thomann, S. Tu, S. Weber, and E. Erdem, Phys. Rev. B 86, 014113 (2012).

${ }^{23}$ J. Szczytko, A. Twardowski, M. Palczewska, R. Jablonski, J. Furdyna, and H. Munekata, Phys. Rev. B 63, 085315 (2001).

${ }^{24}$ N. S. Averkiev, A. A. Gutkin, E. B. Osipov, and M. A. Reshchikov, Sov. Phys. Solid State 30, 438 (1988).

${ }^{25}$ V. L. Korenev, I. A. Akimov, S. V. Zaitsev, V. F. Sapega, L. Langer, D. R. Yakovlev, Yu. A. Danilov, and M. Bayer, Nature Commun. 3, 959 (2012).

${ }^{26}$ J. Schneider, U. Kaufmann, W. Wilkening, M. Baeumler, and F. Köhl, Phys. Rev. Lett. 59, 240 (1987).

${ }^{27}$ C. R. Pidgeon, D. L. Mitchell, and R. N. Brown, Phys. Rev. 154, 737 (1967).

${ }^{28}$ C. Śliwa and T. Dietl, Phys. Rev. B 78, 165205 (2008).

${ }^{29}$ Z. Liliental-Weber, J. Jasinski, and J. Washburn, J. Cryst. Growth 246, 259 (2002).

${ }^{30}$ S. V. Ivanov, T. V. Shubina, V. N. Jmerik, V. A. Vekshin, P. S. Kop'ev, and B. Monemar, J. Cryst. Growth 269, 1 (2004). 